\title{
Imaging Features of Acute Encephalopathy in Patients with COVID-19: A Case Series
}

\author{
(D) S. Kihira, (D) B.N. Delman, (D) P. Belani, (D) Stein, (D) A. Aggarwal, (D) B. Rigney, (D). Schefflein, (D) A.H. Doshi, and (D) P.S. Pawha
} \& $\mathrm{O}$ 트

\section{ABSTRACT}

SUMMARY: Coronavirus disease 2019 was declared a global pandemic by the World Health Organization on March 11, 2020. There is a scarcity of data on coronavirus disease 2019-related brain imaging features. We present 5 cases that illustrate varying imaging presentations of acute encephalopathy in patients with coronavirus disease 2019. MR features include leukoencephalopathy, diffusion restriction that involves the GM and WM, microhemorrhages, and leptomeningitis. We believe it is important for radiologists to be familiar with the neuroradiologic imaging spectrum of acute encephalopathy in the coronavirus disease 2019 population.

ABBREVIATIONS: COVID-19 = coronavirus disease 2019; SARS-CoV = Severe Acute Respiratory Syndrome coronavirus

C oronavirus disease 2019 (COVID-19) is a clinical manifestation of Severe Acute Respiratory Syndrome coronavirus (SARS-CoV) 2 infection. This virus was first detected in Wuhan, China, in December 2019, ${ }^{1}$ and, by March 11, 2020, it had been declared a global pandemic by the World Health Organization. ${ }^{2}$ Recent studies have demonstrated encephalopathic symptoms in up to $36 \%$ of hospitalized patients with COVID-19, including altered mental status, confusion, and headaches. ${ }^{3}$

There is a scarcity of data on COVID-19-related brain MR features. Poyiadji et $\mathrm{al}^{4}$ suggested a possible link between COVID-19 infection and acute necrotizing encephalopathy, and subsequent case reports described encephalitis in patients with COVID-19. ${ }^{5-7}$ A recent study from Turkey included a range of neuroimaging findings in patients with COVID-19 in the intensive care unit, with cortical signal abnormality and diffusion restriction predominating. ${ }^{8}$ Recently, Radmanesh et $\mathrm{al}^{9}$ reported 2 neuroimaging findings, diffuse leukoencephalopathy and WM microhemorrhages, in patients with COVID-19. Respiratory distress is seen in up to $25 \%$ of patients with COVID-19, ${ }^{10}$ and hypoxia itself can result in leukoencephalopathy and microhemorrhages in certain settings. ${ }^{11,12}$ However, the current literature is limited on whether these MR

Received May 13, 2020; accepted after revision June 15

From the Departments of Diagnostic, Molecular and Interventional Radiology (S.K., B.N.D., P.B., A.A., B.R., J.S., A.H.D., P.S.P.), and Neurology (L.S.), Icahn School of Medicine at Mount Sinai, New York, New York.

Please address correspondence to Puneet S. Pawha, MD, Department of Diagnostic, Molecular and Interventional Radiology, Icahn School of Medicine at Mount Sinai and The Mount Sinai Hospital, One Gustave L. Levy Pl, Box 1234, New York, NY 10029; e-mail: puneet.pawha@mountsinai.org

- Indicates open access to non-subscribers at www.ajnr.org

Indicates article with supplemental on-line table.

http://dx.doi.org/10.3174/ajnr.A6715 findings in patients with COVID-19 are due to primary pathology or secondary complications from hypoxia.

We have encountered MR findings of encephalopathy among 5 patients with COVID-19 during the surge of disease in New York City from late March to late April 2020. As COVID-19 continues to grow on a global scale, it is important for radiologists to be familiar with varying presentations of acute encephalopathy that may be encountered in patients with COVID-19 (On-line Table).

\section{CASE 1}

A 59-year-old woman with no known medical history presented with respiratory distress (40\% on room air and required intubation in the emergency department, and, subsequently, was found to be positive for COVID-19 on reverse transcriptase-polymerase chain reaction nasal swab test result. Her family confirmed acute disorientation on the day of presentation. Her hospital stay was complicated by non-ST-elevation myocardial infarction and focal seizures. A CT of the head was unremarkable; however, subsequent MR imaging of the brain (Fig 1) on day 7 of intubation demonstrated patchy and confluent areas of restricted diffusion and FLAIR hyperintensity that involved the cerebral cortices and WM (Fig $1 A$ ), with involvement of the basal ganglia, splenium of corpus callosum (Fig 1B, $-C$ ), and pons (Fig 1D). A small amount of scattered predominantly frontoparietal leptomeningeal FLAIR hyperintensity was present (Fig $1 A$ ). A few small subcortical and corpus callosum microhemorrhages were noted as well as trace intraventricular hemorrhage. CSF bacterial culture showed no growth over 5 days. There was no elevation of leukocytes or protein in the CSF. The COVID-19 viral load was not detected on CSF analysis. Other etiologies, such as E coli, H Haemophilus, 


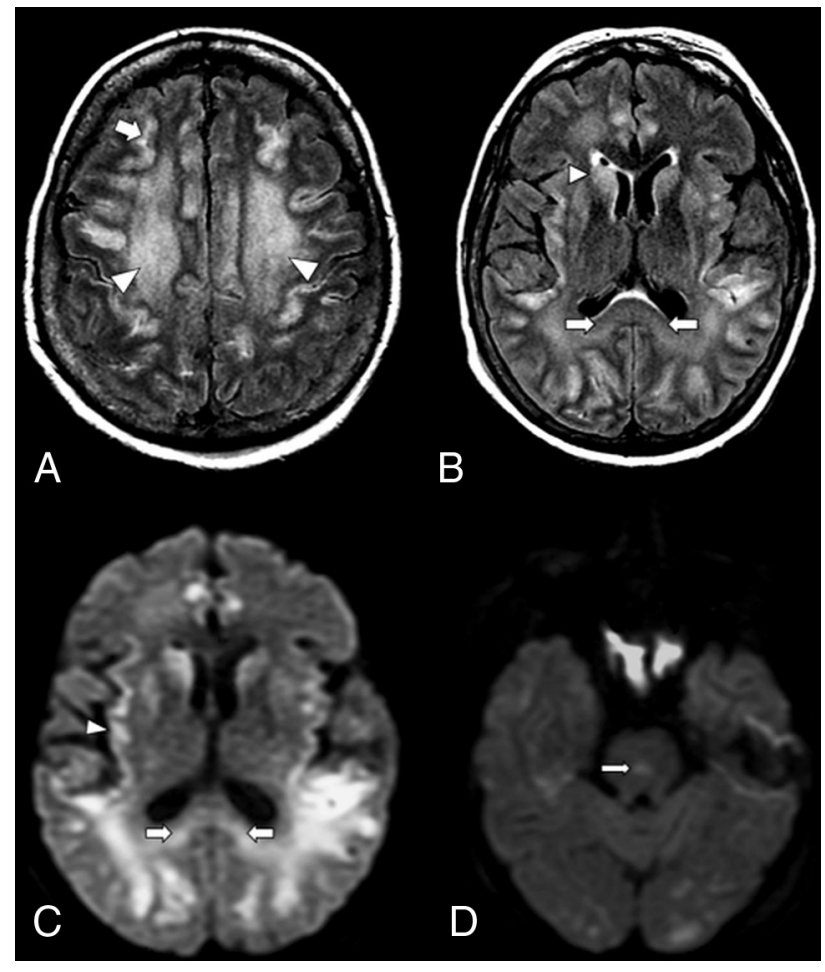

FIG 1. An axial MR image of a 59-year-old woman with COVID-19 who presented with respiratory distress and acute disorientation (case 1). A, FLAIR image demonstrates confluent FLAIR hyperintensity that involves cerebral WM (arrowheads) and patchy involvement of cerebral cortices; a small amount of scattered frontoparietal leptomeningeal FLAIR hyperintensity is noted (arrow). B, FLAIR image shows hyperintensity that involves the splenium (arrows) and basal ganglia (arrowhead). C, DWI demonstrates restricted diffusion that involves the splenium of the corpus callosum (arrows) and the cortex (arrowhead). D, DWI shows a diffusion-restricting focus in the pons (arrow).

N Neisseria, CMV Cytomegalovirus, HSV1 and 2 Herpes simplex virus 1 and 2, and West Nile virus, were not detected on CSF analysis. No oligoclonal bands were present. There was no status epilepticus, discrete anoxic episode, or known toxic and/or metabolic abnormality to account for the imaging findings. The patient is currently undergoing plasma exchange for concern for presumed COVID-19-related autoimmune encephalitis, though hypoxia may also be a contributing factor.

\section{CASE 2}

A 60-year-old man with hypertension and diabetes mellitus type 2 presented with fever, cough, altered mental status, and dyspnea for 2 weeks, and was determined to be positive for COVID-19 on reverse transcriptase-polymerase chain reaction nasal swab test result. The presenting $\mathrm{O}_{2}$ saturation was $92 \%$ on room air. The patient was encephalopathic on presentation, with disorientation and decreased alertness. His hospital stay was complicated by hypoxic respiratory failure, which required intubation and was further complicated by cardiogenic shock attributed to Takotsubo cardiomyopathy. He also developed acute renal failure, which required multiple cycles of dialysis. Although his altered mental status was initially attributed to

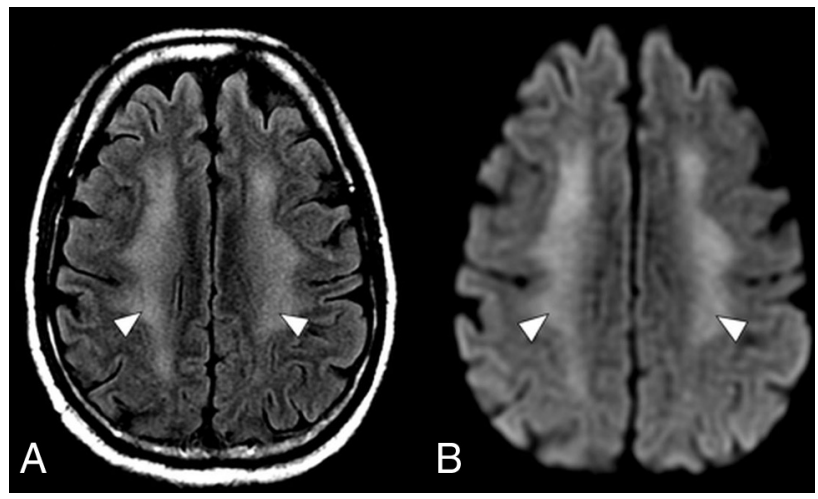

FIG 2. An axial MR imaging of the brain of a 60-year-old man with COVID-19 who presented with disorientation and decreased alertness (case 2). A, A FLAIR image demonstrates diffuse confluent WM hyperintensity with sparing of the subcortical U-fibers (arrowheads). B, DWI shows corresponding restricted diffusion throughout the involved WM (arrowheads).

uremic encephalopathy, he continued to remain altered despite dialysis. A CT of the head was unremarkable except for chronic basal ganglia calcification. Video EEG demonstrated diffuse theta slowing, but no epileptiform discharges. A brain MR imaging ( 2 weeks after intubation) (Fig 2) demonstrated confluent FLAIR hyperintensity and diffusion restriction throughout the cerebral WM, with sparing of subcortical U-fibers. Subsequent CSF analysis was unremarkable and without COVID-19 viral load detection. MR findings and clinical history would be compatible with post-hypoxic leukoencephalopathy; however, because encephalopathy preceded respiratory distress, a component of COVID-19-related leukoencephalitis is also considered.

\section{CASE 3}

A 35-year-old woman at 28 weeks' gestation and diagnosed with pyelonephritis 2 weeks earlier presented with abdominal pain, fever, cough, and decreased alertness, and was positive for COVID-19 on reverse transcriptase-polymerase chain reaction nasal swab test result. $\mathrm{O}_{2}$ saturation was $90 \%$ on room air at presentation. She was being worked up for encephalopathy; however, her hospital stay was complicated by hypoxic respiratory failure, which required intubation, and cardiac arrest a few days later, which required cardiopulmonary resuscitation. After the return of spontaneous circulation, the patient was taken for emergent caesarean section. Her mental status remained poor. A CT of the head was unremarkable. EEG showed generalized slowing compatible with encephalopathy but no electrographic seizures.

MR imaging of the brain 4 days later (day 8 of intubation) (Fig $3 A,-B$ ) demonstrated numerous small foci of susceptibility within the peripheral cerebral subcortical WM and throughout the corpus callosum, most pronounced at the splenium, favored to reflect microhemorrhages. Some foci were linear, which raised the additional but less likely consideration of venous microthrombi. Splenial confluent FLAIR hyperintensity was seen, consistent with edema (Fig 3C); however, no other parenchymal edema was noted. Diffuse prominent sulcal FLAIR hyperintensity was present; however, this is often artifactual in the setting of the 


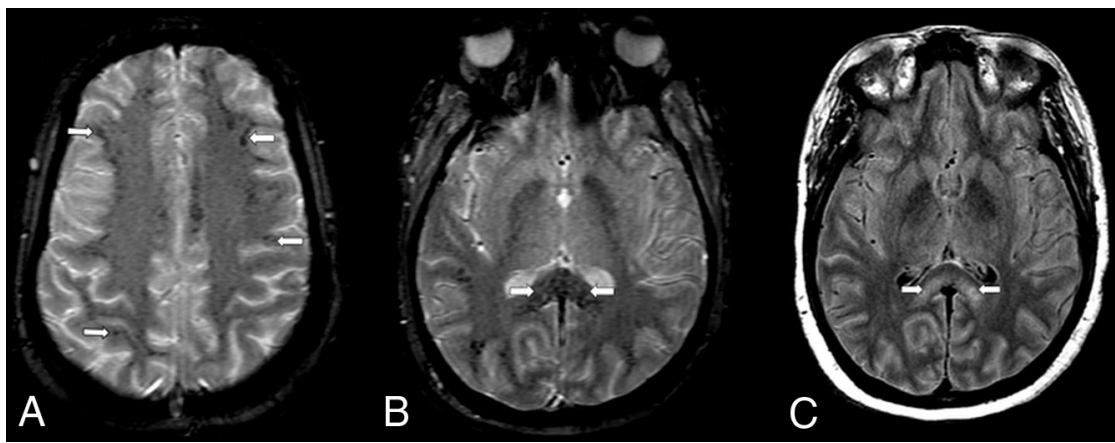

FIG 3. An axial MR imaging of the brain of a 35-year-old woman with COVID-19 who presented with decreased alertness (case 3). A, A gradient recalled-echo (GRE) image demonstrates numerous small foci of susceptibility in the peripheral subcortical WM (arrows). B, A more inferior GRE image shows numerous small foci of susceptibility throughout the corpus callosum, particularly the splenium (arrows). C, A FLAIR image demonstrates confluent bilateral hyperintensity that involves the splenium (arrows).

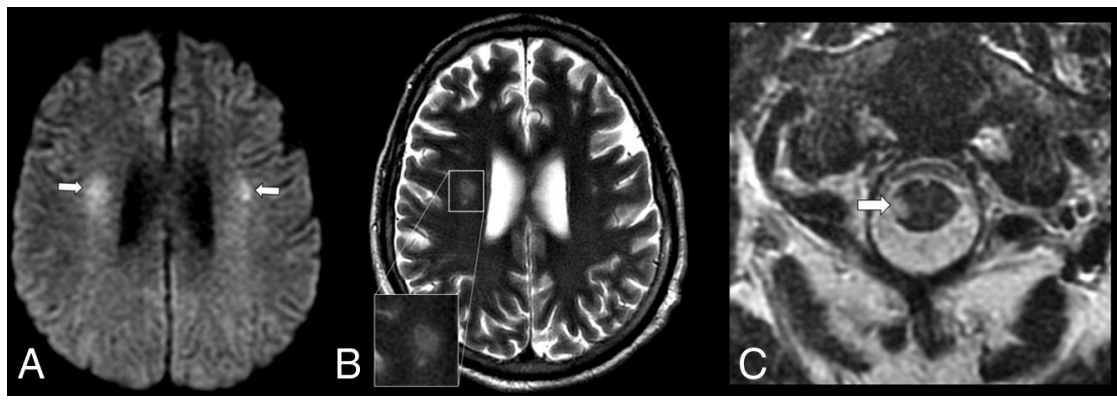

FIG 4. An axial MR imaging of the brain of a 48-year-old man with COVID-19 and with acute flaccid paralysis of the bilateral lower extremities (case 4). A, DWI demonstrates multiple diffusionrestricting foci in the WM, including right greater than left corona radiata (arrows). B, A T2 FSE image shows a "halo" appearance of a dominant right corona radiata lesion (inset), suggestive of demyelination. C, A T2 FSE image demonstrates a focal lesion in the right lateral column of the spinal cord at $\mathrm{Cl}$ (arrow), which corresponds to a diffusion-restricting focus on MR imaging of the brain.

patient receiving high oxygen fraction, which can confound assessment for meningitis. CSF bacterial culture showed no growth for 4 days. There was no elevation of leukocytes or protein in the CSF. Other etiologies, such as $E$ coli, $H$ Haemophilus, $N$ Neisseria, CMV Cytomegalovirus, HSV1 and 2 Herpes simplex virus 1 and 2, and West Nile virus were undetected on CSF analysis. Autoimmune antibodies and oligoclonal bands were also not detected. Testing for the presence of SARS-CoV-2 in the CSF was unable to be performed. A working diagnosis was a hemorrhagic leukoencephalopathy in the setting of active viral infection.

\section{CASE 4}

A 48-year-old man with no known medical history initially presented with nausea, vomiting, and cough, and was found to have COVID-19 on reverse transcriptase-polymerase chain reaction nasal swab test result. His presenting oxygen saturation was $100 \%$ on room air. The first 2 inpatient weeks were complicated by acute respiratory distress syndrome, which required intubation, and acute kidney injury, which required peritoneal dialysis. The patient subsequently became altered, with flaccid paralysis of bilateral lower extremities activating a code stroke. A CT of the head and a CTA were unremarkable, with an ASPECTS of 10.
MR imaging of the brain the following day (week 3 of intubation) (Fig 4A) showed multiple diffusion-restricting and FLAIR hyperintense foci within the WM, specifically in the right centrum semiovale and bilateral corona radiata, left temporal lobe, and left medullary pyramid, and in the spinal cord at $\mathrm{C} 1$. T2 FSE imaging (Fig 4B) showed a halo-like appearance of the dominant lesion in the corona radiata and another, smaller lesion, which favored a demyelinating/inflammatory etiology over infarct. Concurrent MR imaging of the spine (Fig 4C) showed focal T2 hyperintense lesions within the right lateral cord at $\mathrm{C} 1$ (which corresponded to the focal restricted diffusion seen on the MR imaging of the brain) and T5 levels as well as a third lesion in the left lateral column at T5. CSF protein and glucose were elevated to $132 \mathrm{mg} / \mathrm{dL}$ and $105 \mathrm{mg} / \mathrm{dL}$, respectively. There was no oligoclonal band, and the cytopathology result was negative. Given the findings of multiple diffusion restricting lesions limited to WM structures of the brain and cord in the setting of recent COVID-19 infection, a postviral autoimmune demyelinating encephalomyelitis was favored.

\section{CASE 5}

A 41-year-old man with morbid obesity who had previously undergone a gastric sleeve procedure presented with nonproductive cough, shortness of breath, and subjective chills for 4 days. At presentation, oxygen saturation was $95 \%$ on room air. He was found to have COVID-19 on reverse transcriptase-polymerase chain reaction nasal swab test result and associated pneumonia. The hospital course was complicated by hypoxic respiratory failure, which required intubation, and acute kidney injury, which required dialysis. The patient was on ventilation for 3 weeks and was found to be disoriented and decreased in alertness after extubation, which persisted despite several cycles of hemodialysis. A CT of the head performed 4 weeks after intubation was unremarkable, but the subsequent MR imaging demonstrated FLAIR hyperintensity of the globi pallidi and more extensively throughout the cerebral WM, with subcortical U-fiber sparing (Fig 5), with corresponding moderately restricted diffusion. The leading consideration was de-layed post-hypoxic leukoencephalopathy; however, basal ganglia involvement also prompted consideration of encephalitis and metabolic encephalopathy. Postviral demyelination is less likely given the diffuse symmetric MR findings. 


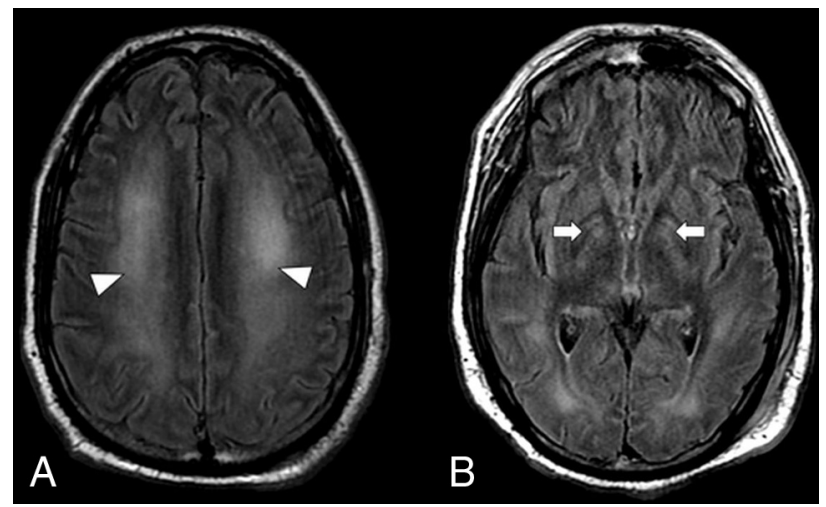

FIG 5. An axial MR imaging of the brain of 41-year-old man with COVID-19 and with persistent disorientation and decreased alertness 1 week after extubation (case 5). A, A FLAIR image demonstrates hyperintensity throughout the cerebral WM (arrowheads). B, A more-inferior FLAIR image demonstrates hyperintensity of the globi pallidi bilaterally (arrows).

\section{DISCUSSION}

The 5 cases presented illustrate varying imaging presentations of acute encephalopathy in patients with COVID-19. There is a scarcity of data on COVID-19-related MR findings of encephalopathy. Differential considerations in all cases included potential complications of the SARS-CoV-2 virus. All 5 patients demonstrate WM signal abnormalities and areas of diffusion restriction. Three of 5 patients demonstrated large confluent and symmetric diffusion restriction in the cerebral WM, with sparing of subcortical U-fibers. This pattern can be seen with delayed post-hypoxic leukoencephalopathy, which is a leading consideration in 2 patients in whom MR imaging was obtained 1-4 weeks after hypoxic events; however, encephalopathy was noted even before hypoxia in 2 of 3 patients with this confluent WM abnormality. Clinically significant hypoxic burden is reported with $5 \%-25 \%$ of patients with COVID-19 and who require ventilation, ${ }^{10,13}$ and even more are presumed to have silent hypoxia before presentation. ${ }^{14}$ This is a confounding variable because hypoxia can result in varying MR findings, for example, those seen in delayed posthypoxic leukoencephalopathy ${ }^{12}$ and high-altitude cerebral edema. ${ }^{11}$ Given the clinically significant hypoxia encountered in patients with COVID-19, we believe that the MR findings in some cases are multifactorial, with a likely frequent component of post-hypoxic leukoencephalopathy. ${ }^{10}$

Results of a few recent studies have suggested COVID-19related neuroimaging findings in patient with encephalopathy. ${ }^{8,9,15}$ Our findings differed from those of Kandemirli et $\mathrm{al},{ }^{8}$ which showed GM involvement in most cases. Radmanesh et $\mathrm{al}^{9}$ reported findings of diffuse leukoencephalopathy and microhemorrhages, which are also present in our series. In addition, there are several important novel findings in our study that may help to understand the broad spectrum of COVID-19-related MR imaging findings. Basal ganglia 2 patients) and pontine (1 patient) involvement of edema and diffusion restriction have not been described in association with COVID-19 in previous studies. In addition, COVID-19related postviral demyelination of the brain and spinal cord is a novel finding.
Viral encephalitis is a sequela of direct viral neuroinvasion and is typically dictated by the receptor binding domain of the virus. Studies in SARS-CoV, whose genomic sequence closely resembles SARS-CoV-2 of COVID-19, ${ }^{16}$ suggest that the pathology involves a cytokine cascade through angiotensin-converting enzyme 2 receptor binding, which leads to blood-brain barrier breakdown. ${ }^{17}$ Angiotensin-converting enzyme 2 is widely expressed in the glial cells and in the brain stem nuclei regulating the cardiorespiratory systems. ${ }^{18}$ Moreover, this cytokine cascade and the direct cytopathic damage by the virus was proposed to cause neurologic disease, such as encephalitis, in susceptible individuals ${ }^{19}$ and was recently described in a subset of patients with COVID-19. ${ }^{20}$ Viral entry into the CNS may result from either hematogenous dissemination or retrograde peripheral nerve propagation within the brain, with the latter appealing for the explanation of anosmia in the COVID-19 population. ${ }^{21}$

Two patients in this case series demonstrated splenial edema, which has also been previously reported in Middle East Respiratory Syndrome coronavirus encephalitis. ${ }^{22}$ The patients in our cases also demonstrated microhemorrhages within subcortical regions and corpus callosum, one extensive and the other mild. This distribution has been described in high-altitude cerebral edema and also in patients who are critically ill ${ }^{11,12}$ and was also described by Radmanesh et $\mathrm{al}^{9}$ as a potential COVID-19related finding. Additional consideration for this appearance includes hemorrhagic leukoencephalitis, possibly even a forme fruste of acute hemorrhagic leukoencephalitis, the autoimmune process that typically has a more fulminant course. In addition, cytokine cascade has been shown to lead to thromboembolic phenomena as a result of a hypercoagulable state, ${ }^{23}$ which could then cause small vessel thromboses and microhemorrhages within the brain. Hemorrhagic foci are also seen in acute necrotizing encephalopathy; however, the patients in our cases show the absence of necrosis and dissimilar distribution. CSF analysis was unremarkable in all cases of suspected possible viral encephalitis in this case study, perhaps not surprising given the low utility of the viral analysis in CSF where levels are typically very low. ${ }^{24}$ However, a negative viral assay does not rule out the diagnosis.

A case of suspected postviral demyelination is included in this case series, presumed to be related to recent COVID-19 infection. Postviral encephalomyelitis or acute disseminated encephalomyelitis is an immune-mediated inflammatory demyelinating disorder that occurs within days to weeks of viral infection. The age of onset remains young in the adult population, with a mean age in the $40 \mathrm{~s}^{25}$ Acute disseminated encephalomyelitis is not the result of tissue invasion by the microbes themselves, unlike in viral encephalitis. Demyelination is often in the cerebral WM and spinal cord, although it may also involve the GM. CSF analysis is nonspecific in patients with acute disseminated encephalomyelitis and may be normal in up to one-third of patients. ${ }^{26}$ Although this series includes the presumptive first report of postviral demyelinating encephalomyelitis from COVID-19, MR imaging features are typical of this entity, and this mechanism would parallel other coronavirus subtypes in which acute disseminated encephalomyelitis has also been described. ${ }^{27}$

A comprehensive delineation of post-hypoxic leukoencephalopathy, postviral autoimmune encephalitis, and infectious 
leukoencephalitis is not possible given our small sample size. Symmetric confluent leukoencephalopathy favors a component of post-hypoxic leukoencephalopathy, and more focal WM lesions (particularly if the spinal cord or the posterior fossa is involved) may suggest postviral autoimmune demyelination. Basal ganglia involvement can raise suspicion for encephalitis in the absence of known causative factors. In addition, we acknowledge that silent hypoxia is associated with COVID-19 infection and would be difficult to measure because the patient would be asymptomatic and not seek medical attention at the time. However, only 1 patient had clinically significantly low oxygen saturation $(<90 \%)$ at the time of presentation, which suggests that encephalopathy may have preceded hypoxia.

Whether these imaging appearances are the sequelae of direct infectious encephalitis, postviral encephalitis, hypoxia, or simply critical illness-related encephalopathy remains under investigation. In some patients with COVID-19, encephalopathy may be multifactorial. This case series serves to raise awareness of varying presentations of presumed encephalitis that may be seen in patients with COVID-19. Knowledge of these neuroimaging features may aid in the detection and early management of neurologic complications in this patient population.

Disclosures: Bradley Delman-UNRELATED: Payment for Lectures Including Service on Speakers Bureaus: Bayer Healthcare, Comments: Internal lectures (no more than 1 per year).

\section{REFERENCES}

1. Huang $\mathrm{C}$, Wang Y, Li X, et al. Clinical features of patients infected with 2019 novel coronavirus in Wuhan, China. Lancet 2020;395:497-06 CrossRef Medline

2. WHO Director-General's opening remarks at the media briefing on COVID-19 - 11. March 2020

3. Weiss $P$, Murdoch DR. Clinical course and mortality risk of severe COVID-19. Lancet 2020;395:1014-15 CrossRef Medline

4. Poyiadji N, Shahin G, Noujaim D, et al. COVID-19-associated acute hemorrhagic necrotizing encephalopathy: CT and MRI features. Radiology 2020;296:E119-E120 CrossRef Medline

5. Moriguchi T, Harii N, Goto J, et al. A first case of meningitis/encephalitis associated with SARS-Coronavirus-2. Int J Infect Dis 2020;94:55-58 CrossRef Medline

6. Sohal S, Mossammat M. COVID-19 presenting with seizures. IDCases 2020;20:e00782 CrossRef Medline

7. Ye M, Ren Y, Lv T. Encephalitis as a clinical manifestation of COVID-19. Brain Behav Immun 2020;88;945-46 CrossRef Medline

8. Kandemirli SG, Dogan L, Sarikaya ZT, et al. Brain MRI findings in patients in the intensive care unit with COVID-19 infection. Radiology 2020 May 8. [Epub ahead of Print] CrossRef Medline
9. Radmanesh A, Derman A, Lui YW, et al. COVID-19 -associated diffuse leukoencephalopathy and microhemorrhages. Radiology 2020 May 21. [Epub ahead of Print] CrossRef Medline

10. Greenland JR, Michelow MD, Wang L, et al. COVID-19 infection: implications for perioperative and critical care physicians. Anesthesiology 2020;132:1346-61 CrossRef Medline

11. Hackett PH, Yarnell PR, Hill R, et al. High-altitude cerebral edema evaluated with magnetic resonance imaging: clinical correlation and pathophysiology. JAMA 1998;280:1920-25 CrossRef Medline

12. Fanou EM, Coutinho JM, Shannon $P$, et al. Critical illness-associated cerebral microbleeds. Stroke 2017;48:1085-87 CrossRef Medline

13. Möhlenkamp S, Thiele H. Ventilation of COVID-19 patients in intensive care units. Herz 2020;45:329-31 CrossRef Medline

14. Xie J, Tong Z, Guan X, et al. Critical care crisis and some recommendations during the COVID-19 epidemic in China. Intensive Care Med 2020;46:837-40 CrossRef Medline

15. Helms J, Kremer S, Merdji H, et al. Neurologic features in severe SARS-CoV-2 infection. N Engl J Med 2020;382:2268-70 CrossRef Medline

16. Lu R, Zhao X, Li J, et al. Genomic characterisation and epidemiology of 2019 novel coronavirus: implications for virus origins and receptor binding. Lancet 2020;395:565-74 CrossRef Medline

17. Zhou P, Yang X-L, Wang X-G, et al. A pneumonia outbreak associated with a new coronavirus of probable bat origin. Nature 2020; 579:270-73 CrossRef Medline

18. Xia H, Lazartigues E. Angiotensin-converting enzyme 2 in the brain: properties and future directions. J Neurochem 2008;107:1482-94 CrossRef Medline

19. Mehta P, McAuley DF, Brown M, et al. HLH Across Specialty Collaboration, UK. COVID-19: consider cytokine storm syndromes and immunosuppression. Lancet 2020;395:1033-34 CrossRef Medline

20. Wu Y, Xu X, Chen Z, et al. Nervous system involvement after infection with COVID-19 and other coronaviruses. Brain Behav Immun 2020;87:18-22 CrossRef Medline

21. Dubé M, Le Coupanec A, Wong AHM, et al. Axonal transport enables neuron-to-neuron propagation of human coronavirus $\mathrm{OC}_{43}$. J Virol 2018;92:e00404 CrossRef Medline

22. Arabi YM, Harthi A, Hussein J, et al. Severe neurologic syndrome associated with Middle East respiratory syndrome corona virus (MERS-CoV). Infection 2015;43:495-501 CrossRef Medline

23. Ma J, Xia P, Zhou Y, et al. Potential effect of blood purification therapy in reducing cytokine storm as a late complication of critically ill COVID-19. Clin Immunol 2020;214:108408 CrossRef Medline

24. Debiasi RL, Tyler KL. Molecular methods for diagnosis of viral encephalitis. Clin Microbiol Rev 2004;17:903-25 CrossRef Medline

25. Sonneville R, Demeret S, Klein I, et al. Acute disseminated encephalomyelitis in the intensive care unit: clinical features and outcome of 20 adults. Intensive Care Med 2008;34:528-32 CrossRef Medline

26. Schwarz S, Mohr A, Knauth M, et al. Acute disseminated encephalomyelitis: A follow-up study of $\mathbf{4 0}$ adult patients. Neurology 2001;56:1313-18 CrossRef Medline

27. Yeh EA, Collins A, Cohen ME, et al. Detection of coronavirus in the central nervous system of a child with acute disseminated encephalomyelitis. Pediatrics 2004;113(pt 1):e73-76 CrossRef Medline 